\section{Disculpe, \\ ¿es aquí el CCMGM? \\ O la sugerencia que el Edificio Diego Portales es un centro cultural}

Excuse me, Is the CCMGM here?

Or the suggestion that the Diego Portales Building is a cultural center
Resumen Durante la madrugada del 29 de septiembre de 2006 cinco equipos de voluntarios, conducidos por la Cooperativa URO1.ORG, realizan una acción señalética para llamar la atención sobre el Edificio Diego Portales. Cinco carteles falsos indican, mediante un isotipo, una flechay un texto, que el Centro Cultural Metropolitano Gabriela Mistral se encuentra cerca. Los carteles son una instrucción, una invitación a moverse, que descubre una historia y activa vínculos que pueden convertirse en motor de cambios programáticos y espaciales.

\title{
Carolina Tobler
}

Palabras Clave Cooperativa URO1.ORG Acción señalétic
UNCTAD III

Edificio Diego Portales

Edificio Diego Portales
Centro Cultural Gabriela Mistral

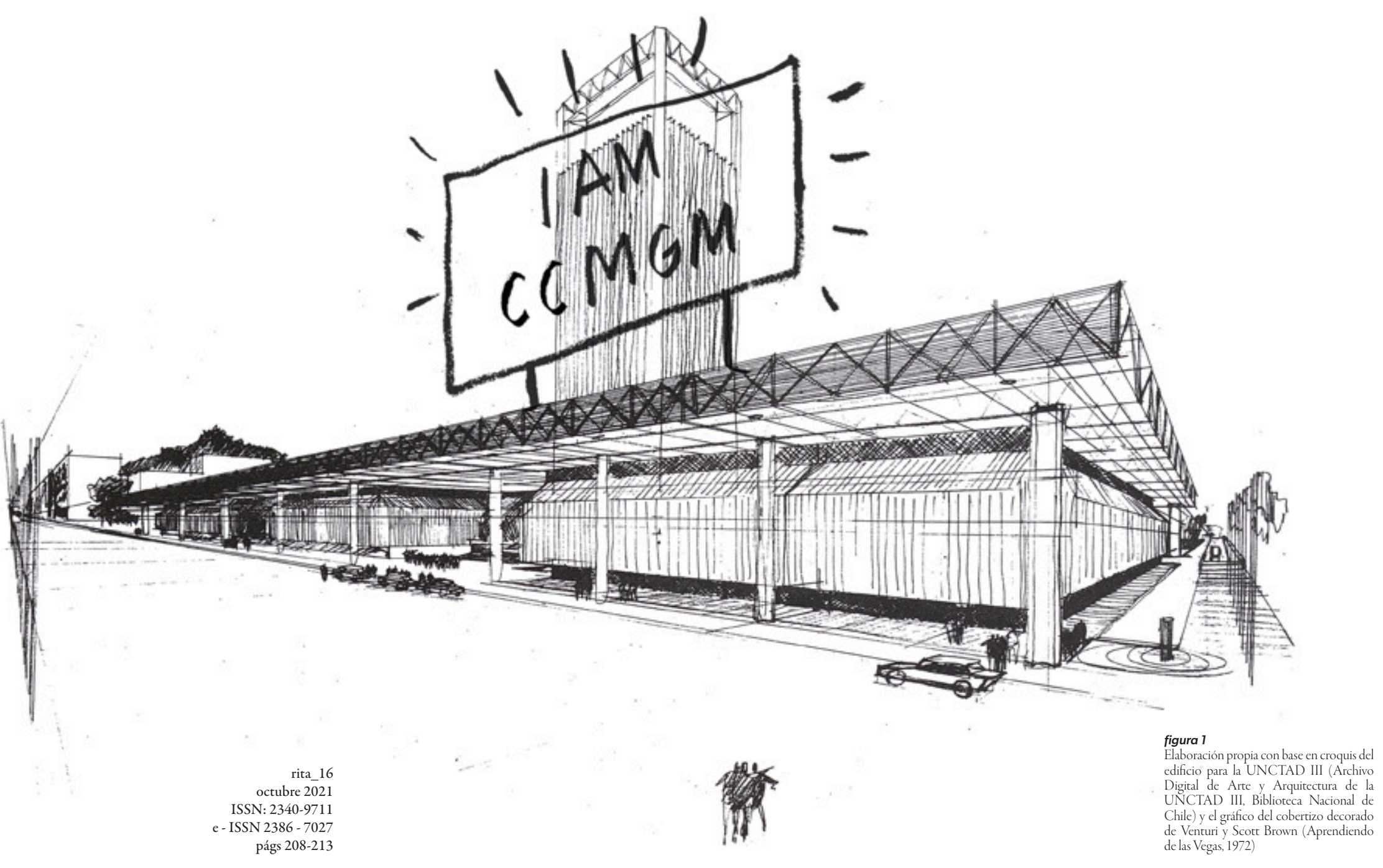


MADRUGADA del

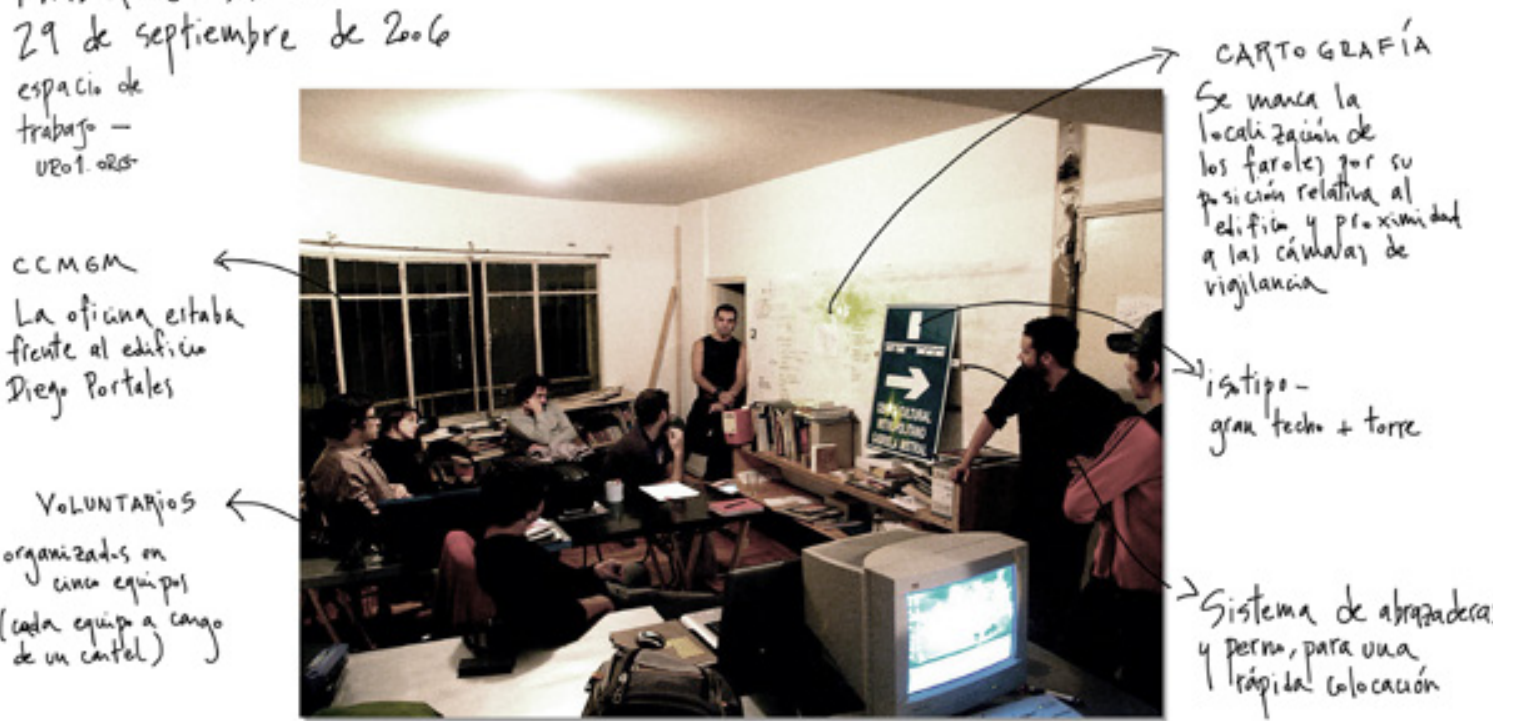

figura 2

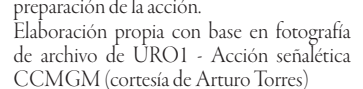

\section{Una historia}

La crónica revisa uno de los muchos episodios de resignificación que vivió el Centro Cultural Metropolitano Gabriela Mistral en sus cincuenta años de historia.

\section{Tres personajes}

\section{El edificio}

El actual Centro Cultural Gabriela Mistral (GAM) registra muchos nombres: Edificio para la Tercera Conferencia Mundial de Comercio y Desarrollo de las Naciones Unidas (UNCTAD III) en 1972, Centro Cultural Metropolitano Gabriela Mistral (CCMGM) / Museo de la Solidaridad entre 1972 y 1973, Edificio Diego Portales de 1973 a 2010.

\section{El colectivo}

La Cooperativa URO1.ORG, formada por Fernando Portal, Nicolás Reboledo, Gabriel Rudolphy, Alejando Soffia y Arturo Torres, fue un laboratorio de conceptos culturales que buscaba con sus proyectos colaborar con los procesos de transformación cultural de la sociedad. Integrantes entrevistados: Nicolás Reboledo (N.R.) y Arturo Torres (A.T.)

\section{La exposición}

URO1.ORG es invitada a la exposición SPAM city, organizada en 2006 por la revista SPAM_arq. SPAM_city reunió los trabajos de arquitectos, artistas y grupos multidisciplinarios, nacionales y extranjeros, que construyeron obras a partir de la observación, exploración e investigación del espacio urbano y las construcciones que las personas realizan al habitarlos. Metropolitano Gabriela Mistral se encuentra cerca. de las Naciones Unidas, UNCTAD III. Curador entrevistado: Pablo Brugnoli (P.B.).

\section{9 de septiembre de 2006}

Durante la madrugada del viernes cinco equipos de voluntarios, conducidos por la Cooperativa URO1. ORG, realizan una acción señalética para llamar la atención sobre el Edificio Diego Portales. Cinco carteles falsos, fabricados por uno de los proveedores que elaboran los letreros oficiales de la ciudad, indican mediante un isotipo, una flecha y un texto, que el Centro Cultural

\section{3 de abril de 1972}

Construida por el gobierno de Salvador Allende en sólo 275 días, se inaugura en Santiago la sede para acoger la Tercera Conferencia Mundial de Comercio y Desarrollo

\section{7 de mayo de 1972}

"En nombre del pueblo y del gobierno de Chile, hago llegar mi emocionada gratitud a los artistas que han donado sus obras para constituir la base del futuro Museo de la Solidaridad (...) que se establecerá luego en el edificio de la UNCTAD III - será el primero que, en un país del Tercer Mundo, por voluntad de los propios artistas, acerque las manifestaciones más altas de la plástica contemporánea, a las grandes masas populares."

\section{0 de diciembre de 1973}

El edificio para la UNCTAD III se convierte de espacio público de libre acceso a centro de operaciones de la dictadura, cambiando su nombre por Edificio Diego Portales. ${ }^{2}$ Sin grandes intervenciones físicas, un duro cambio de programa transforma el edificio en búnker inaccesible y en símbolo de la represión.

N.R.: Existe aún esa idea mítica de recuperar todo lo desaparecido luego del golpe de Estado: las personas, las historias, las obras de arte, el centro cultural.

A.T.: En Madrid conversé con Guillermo Núñez, pintor chileno. Algunas de sus obras estaban en el centro original. Él me comentó que la colección Museo de la Solidaridad se estaba trasladando cuando vino el golpe de Estado. En ese contexto se guardaron algunas obras, pero muchas otras se perdieron. ${ }^{3}$ Para nosotros la mayor pérdida era la de la Colección Solidaridad en términos del proyecto. Entendíamos que era necesario tener un espacio para recuperar ese proyecto.

\section{6 de marzo de 2006}

Se incendia parte del Edificio Diego Portales, reavivando la pregunta acerca de sus posibles futuros.

\section{Mediados de 2006}

N.R.: Nuestra percepción es que hay un legado de la dictadura, de limitación de las libertades públicas. En URO estamos siempre con la pregunta de cómo avanzar para la transformación cultural, de cómo correr el cerco del punto de vista de la cultura, experimentando qué otros espacios de intervención se pueden pensar.

A.T.: La idea de recuperar el CCMGM para nosotros es un proyecto, pero no un proyecto de arquitectura. La acción señalética es parte de la estrategia de ese proyecto. Sí tiene que ver con arquitectura porque hay un edificio implicado, pero se relaciona con la recuperación de una política pública muy avanzada para 1972 (como muchas cosas que pasaron en ese momento).

\section{Comienzos de 2006}

Previo a la acción señalética URO1.ORG desarrolla el documento ¿Qué hacemos con el edificio para la UNCTAD III? donde proponen devolver un espacio público al centro de la ciudad y generar un museo disgregado en el barrio realmente accesible a todos los públicos, así como pasar al edificio las colecciones del Museo de la Solidaridad.

A.T.: Cómo una dictadura convierte un espacio público en espacio privatizado es paradigmático. Y vuelta la democracia (o al menos en transición) los gobiernos parecían haber dado como superado el problema: para nosotros es una situación icónica y simbólica de la permanencia de la dictadura en la ciudad.

N.R.: Inspirados en la idea que el lenguaje construye realidad, pensamos en cómo proyectar esta idea antigua y convertir el Diego Portales en el CCMGM: designemos qué es ese lugar para diseñar el imaginario que el CCMGM ya existe.

\section{1 de octubre de 2006}

Se inaugura la muestra Spam City en el Museo de Arte Contemporáneo de la Quinta Normal.

P.B.:Laexploración/montajeorganizadaporSPAM_arq propone a los colectivos y personas invitados que realicen una intervención original, con un presupuesto no muy grande, pero con total autonomía. Se parte de la idea que el trabajo artístico puede o no representar objetivamente la realidad, pero siempre tiene la capacidad de entrar en conversación con ella, y plantear una relectura. 
URO1.ORG había realizado su intervención callejera unos días antes, creando una ilusión o utopía sobre el edificio Diego Portales: sugieren que el edificio es un centro cultural. El colectivo precisaba salir del espacio del museo y participar de la conversación y políticas públicas, por lo que resulta problemática la posibilidad que su trabajo se transforme en una acción artística: la exposición importa, pero para usarla a su favor. ${ }^{4}$

\section{Agosto de 2007}

A.T.: No sé a ciencia cierta si tuvo alguna relación, pero al mes más o menos se crea la comisión interministerial para el GAM.

Se realiza un concurso para generar un "Gran Centro Cultural para Chile e intervenir el actual Edificio Diego Portales para restituir su vocación pública original y su adecuada integración al contexto urbano donde se emplaza."

\section{4 de septiembre de 2010}

Se inaugura el Centro Cultural Gabriela Mistral (GAM) bajo una concepción cultural y de acceso y uso de los espacios más tradicional que la de 1972. Hay quienes consideran que "si el edificio cumpliera su función, uno podría entrar donde quisiera.

\section{1}

Son muchas las resignificaciones, tanto positivas como negativas, que ha tenido el edificio en sus cincuenta años de existencia. Algunas de ellas no involucran grandes transformaciones físicas: el tijeral de 1971, el vallado de 1973, la apropiación de la fachada durante el estallido social de 2019. ${ }^{7}$ El proyecto de URO1 aparece como una más de esas capas simbólicas que se suma a esa historia. El objeto arquitectónico evidentemente es su soporte, pero quizás estas acciones empujan cuestiones radicales precisamente porque no son permanentes.

\section{9 de septiembre de 2006}

Los carteles colocados por URO1.ORG son una instrucción, una invitación a moverse, que descubre una historia y activa vínculos que pueden convertirse en motor de cambios programáticos y espaciales. La acción señalética, que parte de objetos convencionales, es capaz de "crear nuevos significados dentro del todo y puede usar incluso el cliché para obtener un efecto nuevo."

La insinuación del cambio de programa es claramente política y propone redefinir las instituciones culturales: qué se entiende por cultura, quién accede a la cultura, qué programas pueden vincular la arquitectura a cuestiones discrepantes más allá del arte. En ese sentido, partiendo del edificio abierto que la UNCTAD iba a ser y que hoy no es, la acción confirma cómo "la ciudad puede convertirse en una alucinación de lo que fue, nunca fue, podría haber sido, debería haber sido, no debería haber sido, todavía podría llegar a ser y algunos pueden hacer creer que realmente es."

A.T.: La intención es que la gente (¿un turista desprevenido quizás?) vea los carteles, camine hasta el lugar y pregunte al policía de la puerta: Disculpe, ¿es aquí es el CCMGM?.

\section{Notas}

Presidente Salvador Allende Gossens, "A los artistas del mundo." 2.

Durante el golpe de Estado del 11 de septiembre de 1973 se produjo el bombardeo al Palacio de la Moneda, anulando el uso del edificio

3.

En 2006 muchas obras se encuentran en bodegas y en malas condiciones. Según los cálculos de URO, el valor de mercado de alounas de ellas es mayor a las obras de edificacion necsar 4.

En ese momento diferentes colectivos y arquitectos plantean tácticas similares. Santiago Cirugeda, participante también de la exposición, sostiene:¿Que dicen que lo que hago es arte? Me importa poco. Lo uso a mi favor. (elpais.com/diario/2005/04/02/ 5. 2396767_850215.html.

Bases para el Concurso público internacional de anteproyectos de rquitectura Centro Cultural Gabriela Mistral.

6.

Hugo Gaggero, integrante del equipo de arquitectos del proyecto original. The Clinic, 26.03.2021.(theclinic.cl/2021/03/26/ hugo-gaggero-el-arquitecto-incansable-que-busca-rescatar-lamemoria-del-gam/)

El 18 de octubre de 2019 se produce el estallido social en Chile: la fachada del GAM pasa a funcionar como cartel de denuncia. La fachada se convierte en cartel comunicativo y en símbolo de lo que pasa en la ciudad.

8.

Robert Venturi, Complejidad y contradicción en arquitectura, p 44

Crimson, architectural historians, TooBlessedToBeDepressed, p. 7-8.

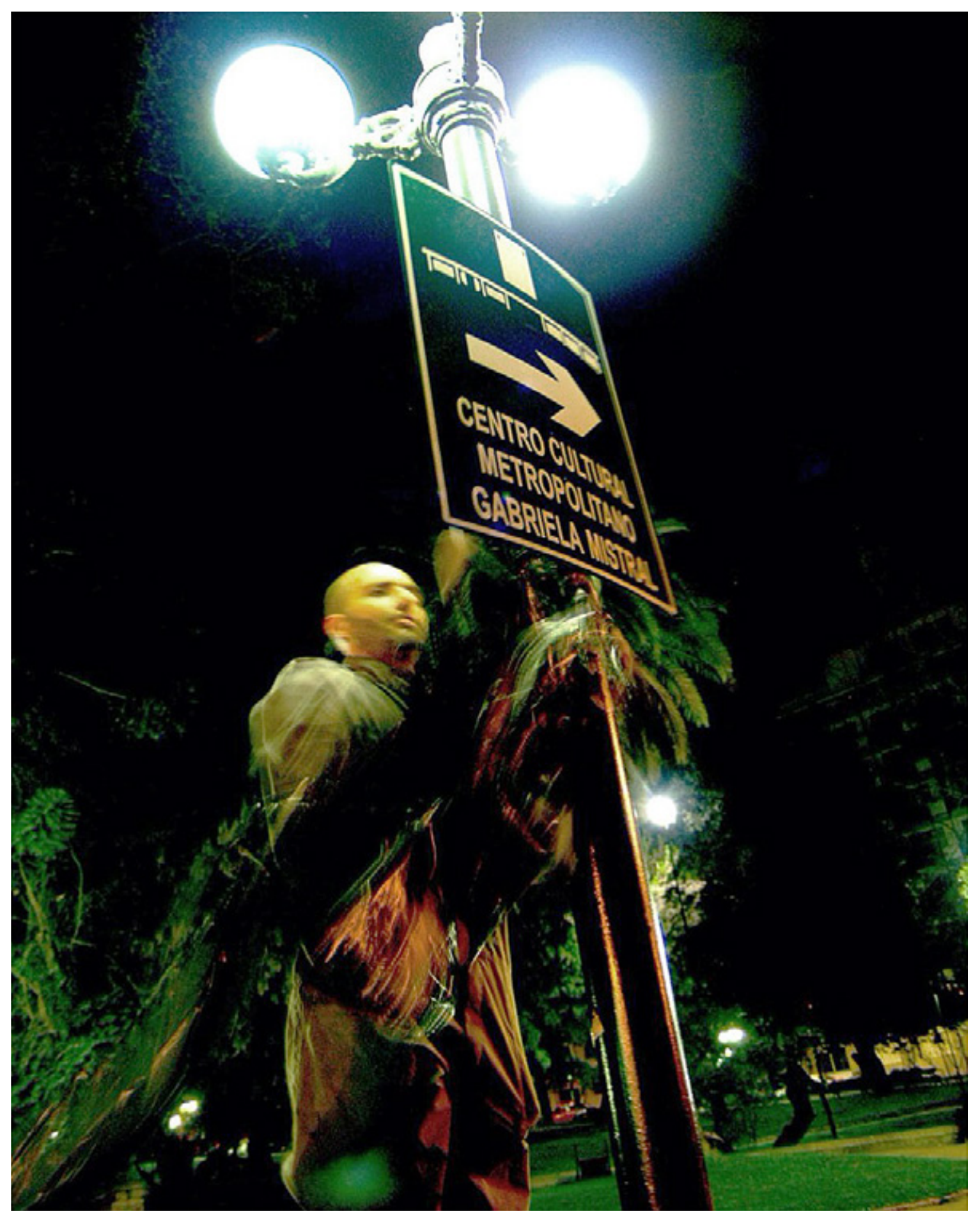

figura 3

Con el cartel colocado, se quita la bolsa negra quelo o cubrey la acción se completa.
Fotografia de archivo de URO1. Acción
señleterica CCMGM (cortesia de Arturo

Carolina Tobler en Arquitectura (PUC, Chile). Docente de historia y taller de proyectos. Investigadora en el Instituto de Estudios Territoriales y Urbanos (FADU). Profesora Visitante (PUC Chile 2021-22). Su oficina, Cotignola-StariccoTobler, representó a Uruguay en la BAL (Pamplona 2019). Ha dictado 